\title{
A Tapping Interface For Finger Movement Training Using Magnetic Sensors
}

\author{
Keisuke Shima*, Toshio Tsuji*, Akihiko Kandori ${ }^{\dagger}$, Masaru Yokoe ${ }^{\ddagger}$ and Saburo Sakoda ${ }^{\ddagger}$ \\ ${ }^{*}$ Graduate School of Engineering, Hiroshima University, Higashi-hiroshima, 739-8527 Japan \\ Email: shima@bsys.hiroshima-u.ac.jp \\ ${ }^{\dagger}$ Fundamental Research Laboratory, Hitachi Ltd., Kokubunji, 185-8601 Japan \\ ${ }^{\ddagger}$ Graduate School of Medicine, Osaka University, Suita, 565-0871 Japan
}

\begin{abstract}
This paper proposes a novel human interface that can be used to operate domestic appliances and game machines. In this system, finger tapping movement is measured by magnetic sensors, and is evaluated by computing five features, such as the finger tapping interval, on a real-time basis. These factors are then discriminated through a probabilistic neural network and allocated as machine operation commands. The user can thus voluntarily operate various machines using finger tapping movements. Experimental results showed that the prototype system developed can learn and classify user movements with a high degree of accuracy (average rates: $98.56 \pm 1.15$ [\%]), and that it can be used to smoothly control appliances and game machines. The possibility of the system supporting finger movement training was also confirmed with three subjects through game operation.

Index Terms-Finger tapping movements, Neural network, Human-machine interface, Magnetic sensor
\end{abstract}

\section{INTRODUCTION}

The number of patients suffering from motor dysfunction due to neurological disorders or cerebral infarction has been increasing in an aging society. In particular, a survey by the Ministry of Health, Labor and Welfare in Japan revealed that the total number of patients with cerebrovascular disease is as high as approximately 137 million people [1]. For patients with such motility function impairment, it is necessary to detect the disease in its early stages by evaluation of motility function and retard its progression through movement rehabilitation training.

Motility function training has been widely applied in clinical environments, and several efficient training methods have been reported [2]-[4]. As an example, Thaut et al. [2] and Enzensberger et al. [3] conducted walking training along with indicated rhythm or melody for patients recovering from strokes or those with Parkinson's disease (PD). Consequently, they confirmed that freezing of gait was decreased, and walking velocity and length of stride were increased. Unfortunately, however, the psychological burden on the subjects was a concern due to the one-sided nature of the training, as the trainees must remain under the constant direction of the therapist and the training system. To enable training to be continued in daily life, it is necessary to develop a method that can lower the psychological burden and allow the trainee to enjoy the training process.

On the other hand, a variety of human interface systems using bioelectric signals such as electromyograms (EMGs) and electroencephalograms (EEGs) as input to control various machines have been developed [5], [6]. Bioelectric signals reflect the internal conditions of the human body and intention regarding body motion. Our research group has also developed several human interfaces that can control domestic appliances, electric wheelchairs and game machines [7]-[9]. Enabling users to operate such machines through voluntary movement can provide an enjoyable and interactive method of motility function training.

The purpose of this study is to realize a system to support enjoyable motility function training for use in daily life, and to this end we propose a novel human interface system using finger tapping movements. Since Holms [10] proved that the rhythm of such movements acts as an efficient index for cerebellar function testing, the quantification of finger tapping movements has already been extensively investigated [11] [14]. Further, the effectiveness of training for PD patients using finger tapping movements was recently discussed [4]. In this study, therefore, we focus on a finger tapping training system that measures finger movements using magnetic sensors [13]. Quantification of finger tapping features, such as the maximum velocity of movement and variation in tapping rhythm, is performed based on medical knowledge. The extracted features are then discriminated using a probabilistic neural network (PNN) and allocated as operation commands for machines such as domestic appliances and a game console. The proposed system not only allows users to train finger movement through operation of these machines, but also enables quantitative evaluation of motility training effectiveness.

This paper is organized as follows: in Section II, the proposed system is described. The details of operation and examples of training experiments performed on healthy subjects are presented in Section III, and the validity of the proposed system is examined. Finally, the paper is concluded in Section IV, and future study plans are discussed.

\section{Finger TAPping Interface System}

The human interface system using finger tapping movements is shown in Fig. 1. This system consists of a magnetic sensor for measuring finger taps, a personal computer (PC) and an infrared (IR) unit [7] for IR communication. The features of the movements measured are computed from the distance between the two fingertips (the fingertip distance), the velocity and the acceleration, and these are discriminated using PNN. The discrimination results and machine operation 


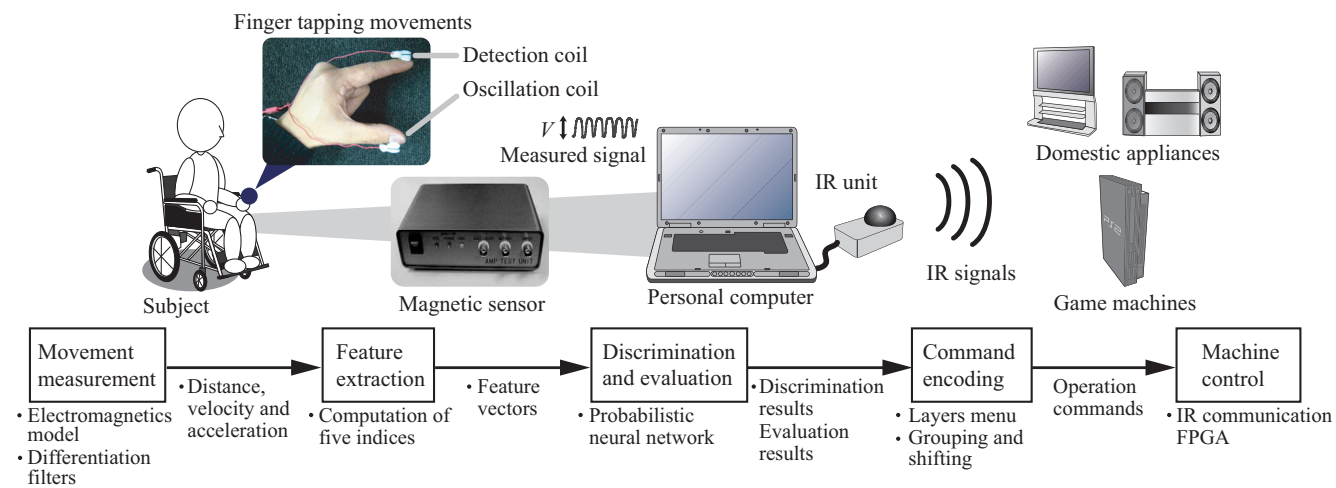

Fig. 1. Concept of the proposed human interface system for finger tapping training

menus are displayed on a monitor, enabling the user to operate domestic appliances and game machines based on the finger tapping movements conducted. The details of each process are explained in the subsections below.

\section{A. Movement measurement}

In this paper, the magnetic sensor developed by Kandori et al. [13] is utilized to measure finger tapping movements. The sensor can output a voltage corresponding to changes in distance between the detection coil and the oscillation coil by means of electromagnetic induction. First, the two coils are attached to the distal parts of the user's fingers, and finger tapping movements are measured. The fingertip distances are then obtained from the output voltage by a calibration model expressed as

$$
\begin{aligned}
d(t) & =\alpha \tilde{V}(t)-\varepsilon \\
\tilde{V}(t) & =V^{-\frac{1}{3}}(t)
\end{aligned}
$$

where $d(t)$ denotes the fingertip distance, $V(t)$ is the measured voltage of the sensors at a given time $t$, and $\alpha$ and $\varepsilon$ are constants computed from the calibration. In the calibration process, $\alpha$ and $\varepsilon$ are estimated using the linear least-square method for $n$ values measured output voltages and the fingertip distances of each subject. The calibration process can reduce the influence of the slope of the coils and modeling errors. Further, the velocity $v(t)$ and acceleration $a(t)$ can be calculated from the fingertip distance $d(t)$ using differentiation filters.

\section{B. Feature extraction}

At the feature extraction stage, the features of finger tapping movements are calculated for quantitative evaluation. The tapping movements of patients with $\mathrm{PD}$, which is characterized by impairment of motor function, have an irregular tapping rhythm whose amplitude gradually decreases due to the deterioration of consecutive motor ability [11]. Moreover, it was discussed that the tapping rhythm of PD patients has smaller maximum amplitudes and velocities but larger variations in tapping interval as compared to that of normal subjects [12],

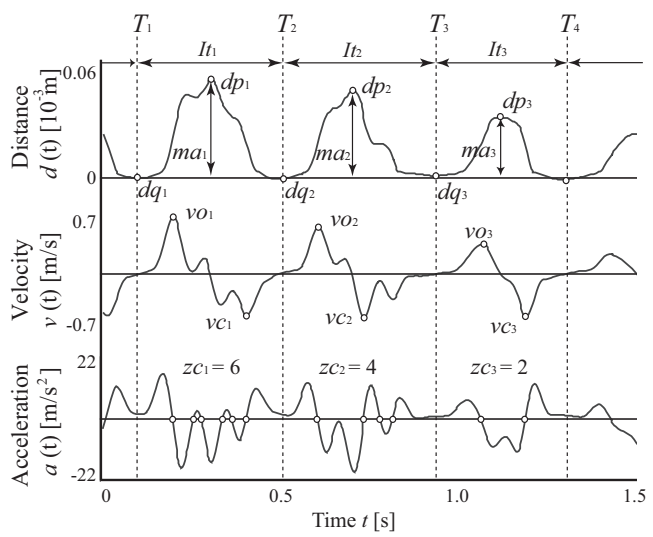

Fig. 2. Examples of the signals measured

[14]. This paper therefore defines five features based on the above observations as follows (see Fig. 2):

1) Maximum amplitude of finger taps $m a_{i}$

2) Finger tapping interval $I t_{i}$

3) Maximum opening velocity $v_{i}$

4) Maximum closing velocity $v c_{i}$

5) Zero-crossing occurrence of acceleration $z c_{i}$

First, the number of fingertip contacts is determined from $d(t), v(t)$, and $a(t)$ for the extraction of a finger tap, and the distance $d_{j}^{\min }$, which satisfies $v(t)=0$ and $a(t)>0$, is calculated from $d(t)$. The instant when the distance $d_{j}^{\text {min }}$ decreases to below the threshold $M^{\text {th }}(\geq \gamma)$ defined by Eq. 3 is defined as the contact time $T_{i}(i=1,2, \ldots, I, I$ is the number of contacts between fingertips).

$$
M^{t h}=\beta\left(\frac{1}{S} \sum_{s=1}^{S} d_{s}^{\max }-\frac{1}{J} \sum_{j=1}^{J} d_{j}^{\text {min }}\right)
$$

Here, $\beta$ and $\gamma$ are constants. $d_{k}^{\max }$ denotes the distance that satisfies $v(t)=0$ and $a(t)<0, K$ is the number of $d_{k}^{\max }$, and $J$ is the number of $d_{j}^{\text {min }}$.

As feature quantities of the $i^{\text {th }}$ tapping, the maximum and minimum amplitude points $\left(d p_{i}, d q_{i}\right)$ between the interval 
$\left[T_{i}, T_{i+1}\right]$ are calculated from the measured fingertip distance $d(t)$, and the maximum amplitudes $m a_{i}=d p_{i}-d q_{i}$ are computed. Further, the finger tapping interval $I t_{i}$, which is the time interval between two consecutive contacts, is applied as $I t_{i}=T_{i+1}-T_{i}$, and the positive and negative maximum velocity points are defined as the maximum opening velocity $v o_{i}$ and the maximum closing velocity $v c_{i}$, respectively. In addition, $z c_{i}$, which denotes the number of zero crossings of the acceleration waveform $a(t)$, is calculated from each interval between $T_{i}$ and $T_{i+1}$, and the zero-crossing occurrences of acceleration $z c_{i}$ are defined as the evaluation value of multimodal movements. Here, the number of zero crossings $z c_{i}$ increases in accordance with the number of extrema of $v(t)$ in a tap movement. As examples, $z c_{3}=2$ implies a smooth tap, while $z c_{1}=6$ or $z c_{2}=4$ would represent a jerky tap (see Fig. 2).

Additionally, the $i^{\text {th }}$ input vector $\boldsymbol{x}(i)=\left[x_{1}(i), x_{2}(i), \ldots\right.$, $\left.x_{5}(i)\right]^{\mathrm{T}}$ is defined as $x_{1}(i)=m a_{i}, x_{2}(i)=I t_{i}, x_{3}(i)=v o_{i}$, $x_{4}(i)=v c_{i}$, and $x_{5}(i)=z c_{i}$ for discrimination of finger tapping movements using PNN.

\section{Discrimination and evaluation}

The extracted features are discriminated for evaluation of finger tapping movements and operation of machines. In this paper, a log-linearized Gaussian mixture network (LLGMN) [15] is used as the PNN. This LLGMN is based on the Gaussian mixture model (GMM) and the log-linear model of the probability density function, and the a posteriori probability is estimated based on GMM by learning. Through learning, the LLGMN distinguishes movement patterns with individual differences and the lag in measurement, thereby enabling precise pattern recognition for bioelectric signals such as EMG and EEG [7]-[9], [15].

In the proposed method, the system first instructs the user to conduct $K$ types of finger tapping movement with different features, such as the amplitude of tapping and the opening velocity. The feature vectors calculated from these movements are then input to the LLGMN as teacher vectors, and the LLGMN is trained to estimate the a posteriori probabilities of each movement. After the training, the system can calculate the similarity between patterns in the user's movements and trained movements as a posteriori probabilities by inputting the newly measured vectors to the LLGMN. In order to prevent discrimination errors, the entropy $E(t)$ (which shows the obscurity of the information) is here calculated from the LLGMN outputs. Since the output $O_{k}(t)$ of the LLGMN represents a posteriori probabilities for each movement $M\left(M=M_{1}, M_{2}, \ldots, M_{K}\right)$, entropy is defined as

$$
E(t)=-\sum_{k=1}^{K} O_{k}(t) \log O_{k}(t)
$$

If $E(t)$ is smaller than discrimination determination threshold value $E_{d}$, the movement with the highest a posteriori probability becomes the result of discrimination. Otherwise, if $E(t)$ exceeds $E_{d}$, discrimination is suspended as obscure movement.
Thus, the finger taps conducted by the user can be classified based on their features of movement using the LLGMN.

Movements can also be evaluated by computation of average value, standard deviation and coefficient of variation of the extracted features at all points of measurement. It is therefore possible to verify the effectiveness of motor function training using the interface system.

\section{Command encoding}

The finger tapping movement of the user $M(M=$ $\left.M_{1}, M_{2}, \ldots, M_{K}\right)$ identified through LLGMN discrimination is allocated as operation command $U\left(U=U_{1}, U_{2}, \ldots, U_{C}\right)$ for each machine. $K$ denotes the number of movements conducted by the user, and $C$ represents the number of commands required to operate machines such as gaming consoles. Here, when the number of $K$ exceeds the total number of $C$, the corresponding estimated movement $M_{k}$ with command $U_{c}$ enables the user to directly execute commands using individual movements. However, since there are limits on the features of finger tapping movements that the user can voluntarily conduct, it is impossible to select all machine operation commands using movement $M_{k}$.

For control of domestic appliances, therefore, operation commands are arranged in a hierarchical structure to enable a range of operations by repeating the commands of execution and selection [7]. With this method, if two patterns (such as menu changes and menu selections) can be distinguished, the system can be operated appropriately.

An example of the interface screen based on GUI function for domestic appliances is shown in Fig. 3, and indicates that the screens of the three hierarchies are layered. Each hierarchy is displayed as one screen. The screen, which is suitable for use in living environments, is designed for intuitive operation. There are several selectable areas on the screen. The user can move from an upper hierarchy to a lower hierarchy by choosing the desired area, and the intended operation is then performed. Fig. 3 shows the process to turn on a television set using the two interface operations of execution and selection.

On the other hand, in the case of game operation, commands are grouped and selected using movements. When the number $K$ of the user movements and the required number $C$ of commands are given, all commands are divided into $G$ groups with $(K-1)$ commands $(K \geq 2)$. The number $G$ becomes

$$
G=\operatorname{ceil}[C /(K-1)]
$$

where ceil $[y]$ is a function giving the minimum integer equal to or larger than real number $y$. The commands included in the group are freely configurable by the user, and can be set up in-line, e.g. increasing the number of commands based on the game machine in order to configure the same commands for multiple groups. The group can be changed using the remaining one of $K$ movements allotted to each group. Based on the above techniques, the user changes groups and selects commands by repeating $K$ movements.

Figure 4 shows an example of a GUI for game operation. This GUI uses a format similar to that of a game control 


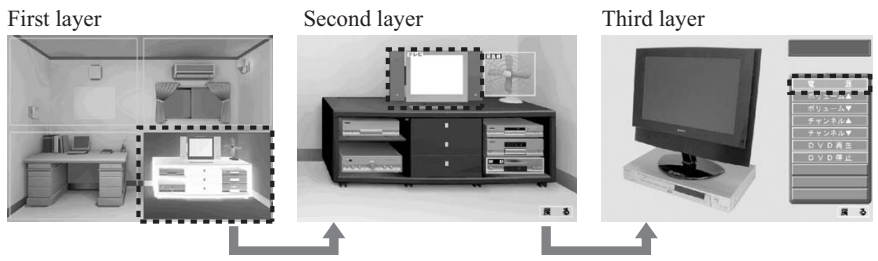

Fig. 3. GUI for domestic appliances

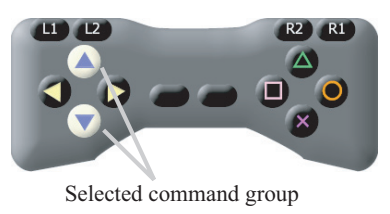

Fig. 4. GUI for game machines pad, and the selected command group is displayed inverted. The user can therefore operate the game machine by shifting groups and selecting commands while watching the graphics on a monitor.

\section{E. Machine Control}

In general, since domestic appliances can be operated using IR communication, an IR transmitter and receiving unit [7] is utilized for the operation of each machine in this system. For domestic appliance operation, the IR signals corresponding to each command are set to the system in advance, and the user then controls each machine through selection of commands using finger tapping movements. In order for the IR unit to support the IR learning function [7], the IR signals of each appliance can be registered and deleted.

On the other hand, since gaming communication protocols differ from machine to machine, the system must be changed as needed. The game machine control circuit is therefore configured as a field-programmable gate array (FPGA) for easy reconstruction [8]. The FPGA, which is a large-scale integrated circuit (LSI), electrically reconfigures the internal circuit by rewriting the program. Less time is taken to implement the targeted circuit than through an application-specific integrated circuit (ASIC), allowing the program to be redesigned. In this system, a generation circuit to issue control signals corresponding to the selected command and a communication circuit to communicate with the game machine are implemented on an FPGA. The generation circuit uses a look-up table (LUT) to pre-store the control signals in the memory, to match the selected commands to the address in the memory, and to generate the required signal. The communication circuit includes the protocol of the individual game machine, and the control signals generated are sent to the machine according to IR signals received from the IR receiver attached to the FPGA.

\section{EXPERIMENTS}

To verify the validity of the proposed interface, we developed a prototype system and conducted experiments involving discrimination of finger tapping movements, operation of domestic appliances and a game machine, and finger tapping training using the proposed prototype. The control circuit of the game machine in the prototype is designed using an evaluation board (RC100, Celoxica) on which the FPGA (XC2S2005FG456) is mounted, and the circuit is described using VerilogHDL. The operation frequency of the circuit is $2.5[\mathrm{MHz}]$, and the control signal bit width stored in the memory is 16 bits.

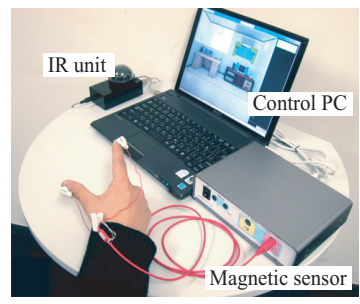

(a) Prototype system developed

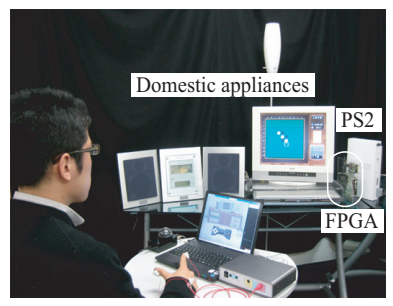

(b) An operation scene using the prototype system
Fig. 5. Photographs of the prototype system and an operation scene

The LUT and communication circuits are implemented based on the communication protocol of the PlayStation 2 (Sony Computer Entertainment Inc., PS2), which the user operates using finger tapping movements.

In the experiments, the subjects (three healthy males, A-C, 23-25 years old) were directed to assume a sitting posture at rest. The coils were attached to the distal parts of the first finger and the index finger as shown in Fig. 1. The magnetic sensor was calibrated using three output voltages and fingertip distances $(0.02,0.03,0.09 \mathrm{~m})$ (Eq. 1). The parameter for determining the contact time of the fingertips was $\beta=0.1$, with a measurement sampling frequency of $100[\mathrm{~Hz}]$. The game used in the experiment was Othello (SUCCESS Corporation), and consent was obtained from all subjects. Figure 5 shows (a) the prototype developed and (b) the operation scene of Othello using the prototype.

\section{A. Operation Experiments}

To examine the effects of discrimination of finger tapping movements, discrimination experiments were conducted using finger taps measured from all subjects. In the experiments, the subjects were asked to conduct two types of movement with low and high velocities $(K=2)$ during a fixed time. For LLGMN learning, 20 sets of feature vectors extracted from these movements were randomly selected, and a total of 40 sets of patterns were used as teacher vectors. The subjects were then asked to repeat two types of movement alternately, and the tapping was measured during a 20 -second period. There were five trials, and the discrimination determination threshold was $E_{d}=0.1$.

An example of the results of finger tapping movement discrimination with subject $\mathrm{A}$ is shown in Fig. 6. This shows the plot of the measured fingertip distance $d(t)$ using a magnetic sensor, velocity $v(t)$, acceleration $a(t)$ waveforms 


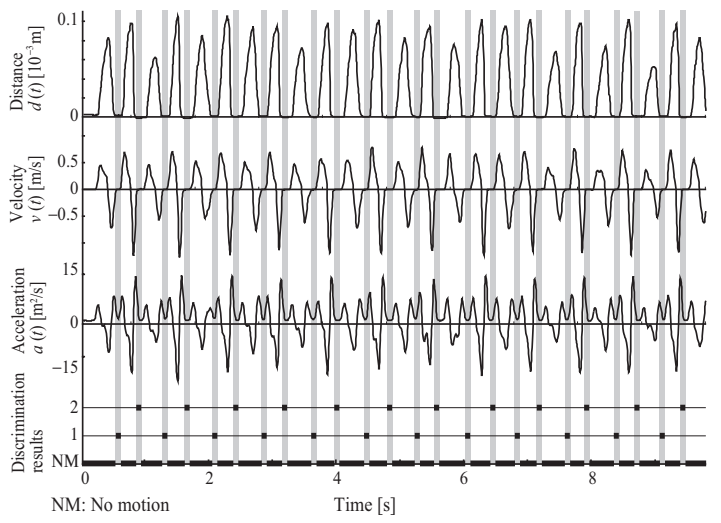

Fig. 6. An example of discrimination results (Subject A)

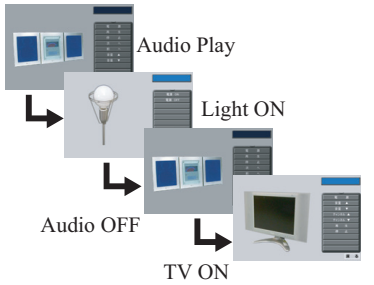

(a) Home electric appliances operation

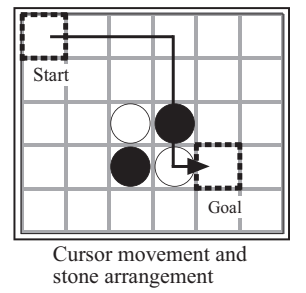

(b) Game operation
Fig. 7. The target tasks in the experiments

and the discrimination results. The figure describes the results of the data measured during the interval $0-10 \mathrm{~s}$. The shaded area indicates the contact time of the fingertips, and No motion $(\mathrm{NM})$ in the discrimination results represents periods of no motion using Eq. 3. From the figure, it was confirmed that the subject performed low- and high-velocity movements iteratively, and that the movements were discriminated accurately by the system. The average discrimination rate of all trials with all subjects was $98.56 \pm 1.15$ [\%].

Experiments with domestic appliances and game operation were also conducted. In these experiments, the subjects were asked to operate the machines by voluntarily performing four types of finger tapping movement $(K=4)$ related to velocity and amplitude. These were $M_{1}$ (low velocity and small amplitude), $M_{2}$ (low velocity and large amplitude), $M_{3}$ (high velocity and small amplitude) and $M_{4}$ (high velocity and large amplitude). Instructions were given to operate each machine as presented in Fig. 7. Figure 8 shows examples of the results of operation ((a) domestic appliance operation; (b) game operation), and includes fingertip distance $d(t)$, velocity $v(t)$, acceleration $a(t)$ waveforms, discrimination results, layers of menu and command groups, and selected commands. The shaded area indicates the contact time of the fingertips. It should be noted that two movements $\left(M_{1}\right.$ and $\left.M_{2}\right)$ were used for operation of domestic appliances, and four $\left(M_{1}\right.$ to $M_{4}$ ) were used for game operation. Here, the changing of groups by game operation commands was decided based

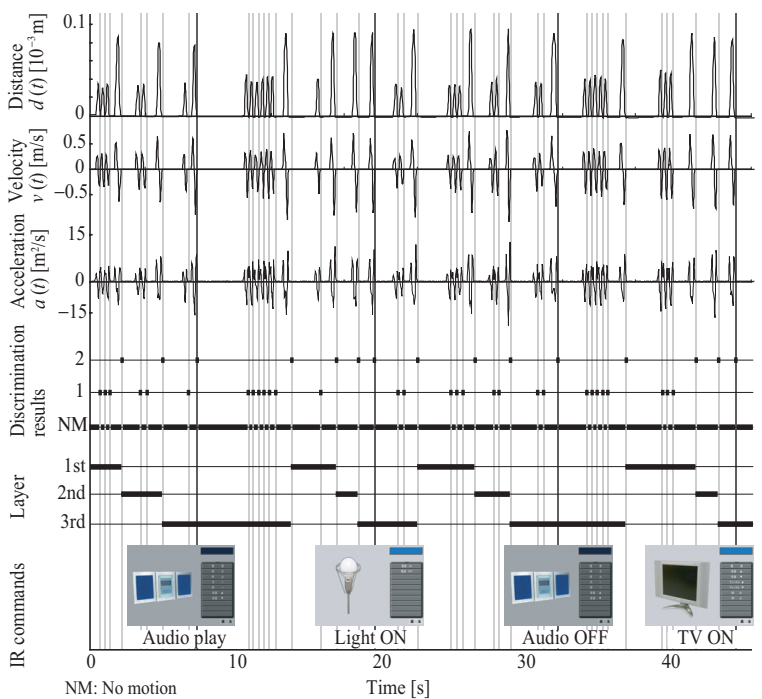

(a) An example of home electric appliances operation

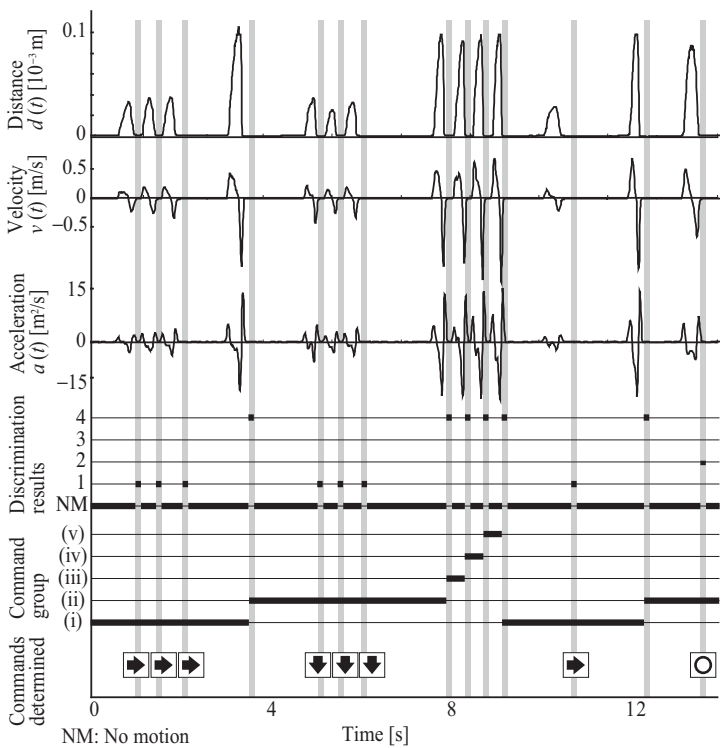

(b) An example of game operation

Fig. 8. An example of operations using the four types of finger tapping movements (Subject A)

on Fig. $9(C=14)$. Fig. 8 shows that the subjects could operate each machine using finger tapping movements with different velocities and amplitudes. We therefore concluded that the subjects were able to voluntarily conduct finger taps and operate the machines as instructed.

\section{B. Example of the training experiments}

To identify the effectiveness of the proposed interface for motility training, training experiments were conducted on all subjects. After a brief trial, finger tapping movement was 


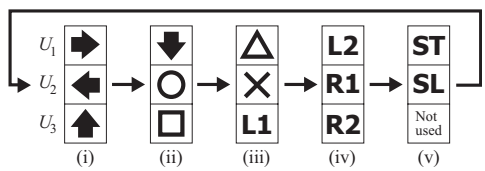

Fig. 9. The command groups in the operation experiments

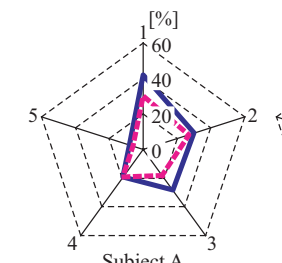

Subject A

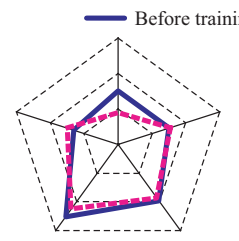

Subject B

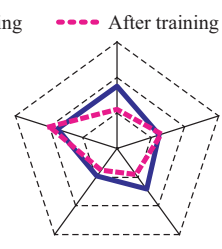

Subject C

$1: \mathrm{CV}$ of maximum amplitude $\quad 4: \mathrm{CV}$ of finger tapping interval

$2: \mathrm{CV}$ of maximum opening velocity $5: \mathrm{CV}$ of zero-crossing occurrence of acceleration

$3: \mathrm{CV}$ of maximum closing velocity $\mathrm{CV}$ : Coefficient of variation

Fig. 10. Experimental results of training with each subject

measured for $30 \mathrm{~s}$ with the instruction to move the fingers while maintaining values for the maximum amplitude of finger taps, the finger tapping interval, the maximum opening velocity and the maximum closing velocity. The average of each value and half the average value of the maximum amplitude of finger taps were then used as teacher vectors of class 1 and 2 respectively, and the LLGMN was trained $(K=2)$. The subjects could therefore operate the game using two types of movement $\left(M_{1}\right.$ : large amplitude of finger taps; $M_{2}$ : small amplitude). In other words, the subjects had to reproduce two types of trained movement for game operation. Further, they were instructed to play one game of Othello, and the movements were measured again after the game. The discrimination determination threshold was $E_{d}=0.1$.

Figure 10 shows the experimental results, and plots each subject's coefficient of variation (CV) of each feature measured for $30 \mathrm{~s}$ before and after game operation. It is observed that the CVs of each value after the game are smaller than those before it. As the results indicate, the proposed interface system is feasible for use in motility function training of finger tapping movements through game machine and domestic appliance operation.

\section{CONCLUSION}

A finger tapping interface that enables operation of game machines and domestic appliances for rehabilitation training is proposed in this paper. The system involves the computation of five features measured from finger movements using magnetic sensors, and the movements are discriminated and allocated as operation commands for each machine.

The results obtained in the experiments using the prototype developed are summarized below.

- The proposed system was able to discriminate finger tapping movements voluntarily conducted by the subjects with high accuracy. The average discrimination rate was $98.56 \pm 1.15[\%]$ with all subjects.

- The subjects were able to operate the domestic appliances and game machine as instructed.

- In the case of finger movement training, the coefficient of variance in the features of each finger tap was reduced in comparison to before game-operation training.

In future research, we would like to investigate the effects of training for patients with motility function impairment such as cerebrovascular disease using the proposed interface with an increased number of subjects, and to discuss adjusting the complexity of control tasks in domestic appliances and game machines for effective training. We also plan to study a quantitative evaluation system based on finger tapping movement analysis for diagnosis support.

\section{ACKNOWLEDGMENT}

This study was supported in part by a Grant-in-Aid for Scientific Research $(19 \cdot 9510)$ from the Research Fellowships of the Japan Society for the Promotion of Science for Young Scientists.

\section{REFERENCES}

[1] Statistics and Information Department, Minister's Secretariat, Ministry of Health, Labour and Welfare: Patient survey, http://www.mhlw.go.jp/ toukei/saikin/hw/kanja/05/index.html (in Japanese)

[2] M.H. Thaut, G.C. McIntosh, R.R. Rice: Rhythmic facilitation of gait training in hemiparetic stroke rehabilitation, Journal of Neurological Sciences, vol. 151, pp. 207-212, 1997.

[3] W. Enzensberger, U. Oberlander, K. Stecker: Metronomtherapie bei Parkinson-Patienten, Der Nervenarzt, vol. 68, pp. 972-977, 1997.

[4] M.F. del Olmo, P. Arias, M.C. Furio, M.A. Pozo, J. Cudeiro: Evaluation of the effect of training using auditory stimulation on rhythmic movement in Parkinsonian patients-a combined motor and $\left[{ }^{18}\right.$ F]-FDG PET study, Parkinsonism and Related Disorders, vol. 12, pp. 155-164, 2006.

[5] R. Barea, L. Boquete, M. Mazo and E. Lopez, "System for Assisted Mobility using Eye Movements based on Electrooculography," IEEE Trans. on Neural Systems and Rehabilitation Engineering, vol. 10, no. 4, pp. 209-218, 2002.

[6] K. Tanaka, K. Matsunaga, H. O. Wang, "Electroencephalogram-Based Control of an Electric Wheelchair," IEEE Trans. on Robotics, vol. 21, no. 4 , pp. 762-766, 2005.

[7] K. Shima, R. Eguchi, K. Shiba, and T. Tsuji, "CHRIS: Cybernetic Human-Robot Interface Systems," Proceedings of 36th International Symposium on Robotics, WE1C3, 2005.

[8] K. Shima, M. Okamoto, N. Bu, and T. Tsuji, "Novel Human Interface for Game Control Using voluntarily Generated Biological Signals," Journal of Robotics and Mechatronics, vol. 18, no. 5, pp. 626-633, 2006.

[9] O. Fukuda, T. Tsuji, M. Kaneko and A. Otsuka, "A Human-Assisting Manipulator Teleoperated by EMG Signals and Arm Motions," IEEE Trans. on Robotics and Automation, vol. 19, no. 2, pp. 210-222, 2003.

[10] G. Holmes, "The symptoms of acute cerebellar injuries due to gunshot injuries," Brain, vol. 40, no. 4, pp. 461-535, 1917.

[11] S. Sakoda, K. Akazawa, K. Abe, and M. Yokoe, "A new dynamic measurement for neurological disorders," Advanced medicine of neurology, Institute of Advanced Medical Technology, pp. 288-293, 2004. (in Japanese)

[12] R. Agostino, A. Curra, M. Giovannelli, N. Modugno, M. Manfredi, A. Berardelli, "Impairment of individual finger movements in Parkinson's disease, "Movement Disorders, vol. 18, no. 5, pp. 560-565, 2003.

[13] A. Kandori, M. Yokoe, S. Sakoda, K. Abe, T. Miyashita, H. Oe, H. Naritomi, K. Ogata, K. Tsukada, "Quantitative magnetic detection of finger movements in parients with Parkinson's disease, "Neuroscience Reseach, vol. 49, no. 2, pp. 253-260, 2004.

[14] R. Okuno, M. Yokoe, K. Akazawa, K. Abe, S. Sakoda, "Finger taps acceleration measurement system for quantitative diagnosis of Parkinson's disease, " Proceedings of the 2006 IEEE International Conference of the Engineering in Medicine and Biology Society, pp. 6623-6626, 2006.

[15] T. Tsuji, O. Fukuda, H. Ichinobe, and M. Kaneko, "A Log-Linearized Gaussian Mixture Network and Its Application to EEG Pattern Classification," IEEE Trans. on Systems, Man, and Cybernetics-Part C: Applications and Reviews, vol. 29, no. 1, pp. 60-72, 1999. 